\title{
Patients with different types of arthritis may be at risk for major depression: results from the National Health and Nutrition Examination Survey 2007-2018
}

\author{
Mi-Yuan Wang ${ }^{1 \#}$, Jie Li ${ }^{2 \#}$, Hong-Ye Peng ${ }^{1 \#}$, Jia Liu ${ }^{1}$, Kai-Lin Huang ${ }^{1}$, Lei Li ${ }^{1}$, Zao-Fang Yan ${ }^{1}$, \\ Zhen-Hai Zhao ${ }^{3}$ \\ ${ }^{1}$ Beijing University of Chinese Medicine, Beijing, China; ${ }^{2}$ College of Traditional Chinese Medicine, Shandong University of Traditional Chinese \\ Medicine, Jinan, China; ${ }^{3}$ Dongzhimen Hospital of Beijing University of Chinese Medicine, Beijing, China \\ Contributions: (I) Conception and design: ZH Zhao; (II) Administrative support: None; (III) Provision of study materials or patients: None; \\ (IV) Collection and assembly of data: MY Wang; (V) Data analysis and interpretation: MY Wang; (VI) Manuscript writing: All authors; (VII) Final \\ approval of manuscript: All authors. \\ "These authors contributed equally to this work. \\ Correspondence to: Zhen-Hai Zhao. Dongzhimen Hospital of Beijing University of Chinese Medicine, Hai Yun Cang on the $5^{\text {th }}$ Zip, Dongcheng \\ District, Beijing 100700, China. Email: linyisd@163.com.
}

Background Arthritis is one of the common causes of physical pain and disability, which often makes patients fall into major depression. However, the correlation between arthritis and major depression, and how different types of arthritis correspond to major depression remain to be explored. The purpose of this study is to explore the relationship between arthritis and major depression.

Methods: Arthritis status was reported by participants themselves, and the Patient Health Questionnaire-9 in National Health and Nutrition Examination Survey (NHANES) was used to evaluate major depression, logistic regression was used to evaluate the relationship between arthritis and major depression.

Results: We analyzed the data of 25,990 adults who participated in the NHANES from 2007 to 2018. Participants with major depression were more likely to be female, Hispanic, smoker, less educated, less recreational activities, poverty-to-income ratio $<5$, coronary heart disease, stroke, cancer or malignant tumor, diabetes, hypertension and higher body mass index (BMI). Arthritis was significantly correlated with major depression $(25.4 \%$ vs. 44.9\%; $\mathrm{P}<0.001)$, even after adjusting for gender, age, race, BMI, PIR, education, marriage, moderate recreational activities, smoking, history of coronary heart disease, stroke, cancer or malignant tumor, diabetes, and hypertension ( $\mathrm{OR}=2.30$, 95\% CI, 2.06-2.56, $\mathrm{P}<0.001)$. Subgroup analysis showed that compared with degenerative arthritis, rheumatoid arthritis (RA), or other arthritis, psoriatic arthritis (PsA) had the greatest influence on major depression patients.

Conclusions: All patients with arthritis, especially PsA, may have the risk of major depression. Psychological intervention necessary for patients with arthritis.

Keywords: Major depression; arthritis; psoriatic arthritis (PsA); National Health and Nutrition Examination Survey (NHANES); cross-sectional study

Submitted Jan 31, 2021. Accepted for publication Apr 08, 2021.

doi: 10.21037/apm-21-279

View this article at: http://dx.doi.org/10.21037/apm-21-279

\section{Introduction}

Arthritis refers to the infections in and around the joints. It is a common clinical chronic systemic inflammatory disease, which is characterized by redness, swelling, heat and pain. Arthritis is a common chronic disabling disease in America. According to the research based on the National Health 
Interview Survey database, about 22.7 percent of adults in the United States have been diagnosed with arthritis by doctors, and 9.8 percent have limited activities, such as difficulty in dressing or going to the toilet, due to arthritis. Arthritis is a common disease not only in the United States but around the world. There are currently 355 million people suffering from arthritis in the worldwide. It is estimated that by 2040, adults diagnosed with arthritis account for $25.9 \%$ of all American adults (1). Arthritis can lead to severe joint pain and dysfunction, resulting in limited movement and decreased quality of life. Arthritis and its limited activity will remain a serious problem in clinical and public health systems.

The most common form of arthritis is osteoarthritis, but arthritis also includes rheumatoid arthritis (RA), psoriatic arthritis (PsA), gout, etc. Osteoarthritis, also known as degenerative arthritis, is a degenerative change, mainly manifested by the degenerative damage of articular cartilage, and the reactive hyperplasia of joint edge and subchondral bone, mainly in the elderly. It is most common among middle-aged and elderly people. While RA, PsA and other types of arthritis are more common inflammatory arthritis, whose pathological manifestations are primarily synovitis and vasculitis. Because of severe joint pain and limited activity, arthritis has a great impact on patients' mental health. A multicenter cross-sectional study showed that patients with knee osteoarthritis were more likely to be depressed, and $10.4 \%$ of these depressed patients suffer from moderate to severe depression (2). Baerwald et al. (3) found that the risk of depression in patients with RA was significantly higher than that of the general population. Depression has adverse effects on the treatment of RA, such as reducing the remission rate and increasing mortality rate. A meta-analysis found that patients with PsA were generally depressed and anxious, with a higher incidence of depression (4), Matcham and his colleagues (5) found that the prevalence rate of major depression among patients with PsA is about $16.8 \%$. Ryu et al. (6) found that RA and degenerative arthritis may have a greater impact on the risk of major depression than other diseases. According to survey statistics, Major Depressive Disorder (MDD) affects about $6 \%$ of adults worldwide every year, and is the second major factor contributing to the burden of chronic disease. In addition, the possibility of suicide among MDD patients is almost 20 times of that in general population. Clinical studies have shown that arthritis patients are more likely to suffer from major depression. However, few large sample studies have confirmed this correlation, and there is a lack of cross-sectional comparisons between different types of arthritis and major depression risks.

This study aims to explore the relationship between arthritis and major depression, as well as the relationship between different types of arthritis and major depression risk through a nationally representative population-based cohort study.

We present the following article in accordance with the STROBE reporting checklist (available at http://dx.doi. org/10.21037/apm-21-279).

\section{Methods}

\section{Study design}

The data used in this study comes from the National Health and Nutrition Examination Survey (NHANES) from 2007 to 2018. NHANES is a national cross-sectional survey which collects nationally representative health and nutrition data, and uses complex and multi-stage probability samples of American civilians and non-institutionalized population to determine the prevalence and risk factors of major diseases in the American population (7). The study was conducted by doctors and experienced medical staff using open online data, including questionnaires, physical examinations and laboratory data (http://www.cdc.gov). In addition, our study conformed to the provisions of the Declaration of Helsinki (as revised in 2013).

\section{Study population}

The history of arthritis was investigated in 35,162 adults ( $\geq 18$ years old). Participants who answer "no" or "don't know", or those who have at most one missing answer to Patient Health Questionnaire-9 (PHQ-9) questions, will be excluded from our analysis $(n=3,702)$. Participants who lack information or have a "no" or "unknown" response to arthritis history will also be excluded $(n=1,822)$. The missing value of other variables is 3,648 . The final sample size included in the analysis was 25,990, of which 7,049 admitted to having arthritis history and 18,941 did not. Figure 1 presented a flow chart of the 2007-2018 NHANES survey.

\section{Measurements and variables}

\section{Definition of primary exposure}

The history of arthritis is determined by a questionnaire, in 


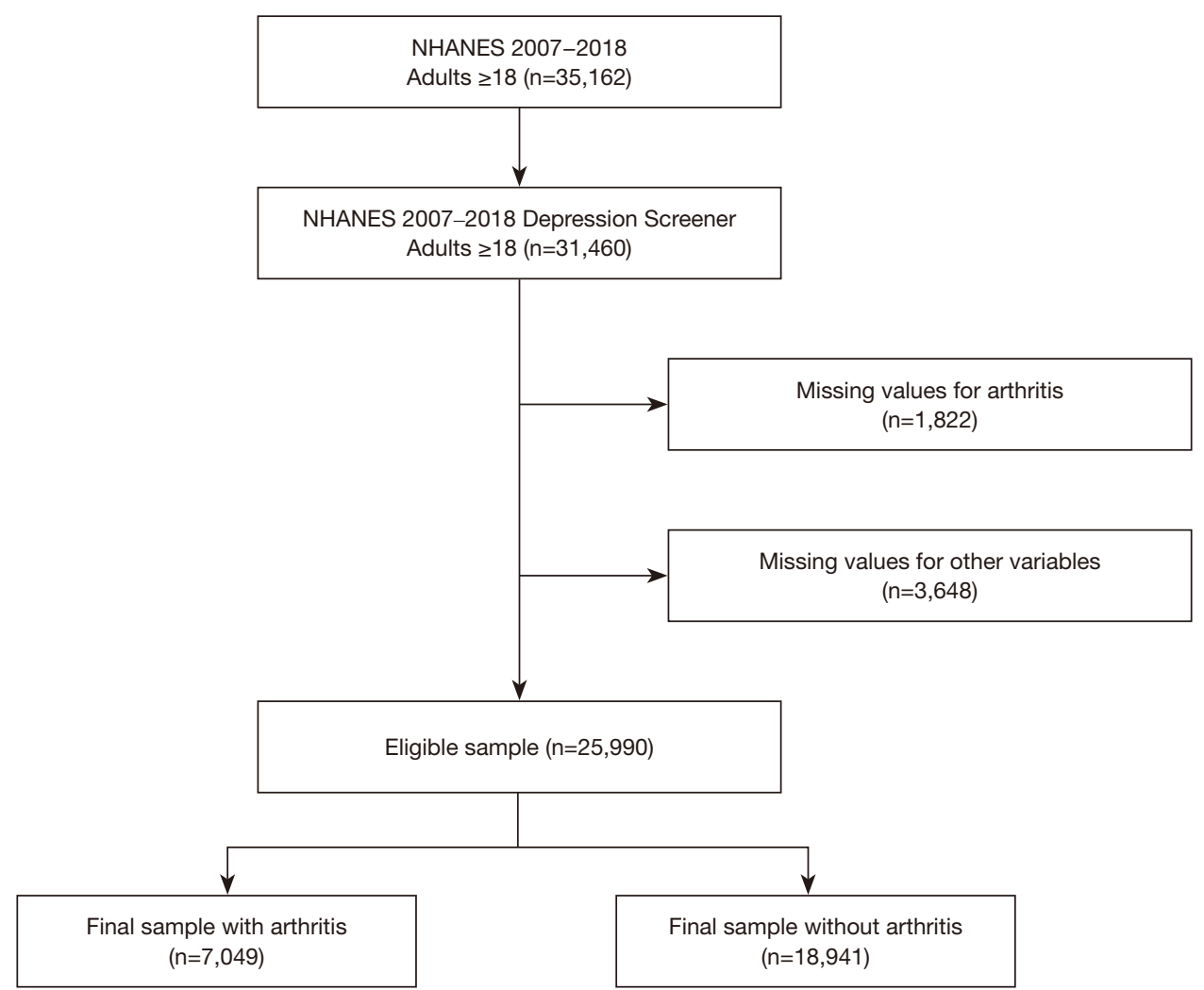

Figure 1 Flowchart of the study sample.

which the patient answers the question "have any doctors or other health experts told you that you have arthritis?" with "yes" or "no". If the answer is "yes", the patient will be further urged to answer "what type of arthritis is this?" "osteoarthritis, degenerative arthritis, rheumatoid arthritis, psoriatic arthritis or other?".

PHQ-9 is a simple and effective self-rating scale for depression, which is based on Diagnostic and Statistical Manual of Mental Diseases formulated by American Psychiatric Society (DSM)-IV. It has good reliability and validity in assisting the diagnosis of depression and evaluating the severity of symptoms. PHQ-9 scores each of the nine DSM-IV criteria as "0" (not at all) to " 3 " (nearly every day) (8). Participants can chose "not at all" (score of 0 ), "several days a week" (score of 1 ), "more than half the days" (score of 2), or "nearly every day" (score of 3) to answer the following nine questions. The total score of PHQ-9 is 27 , and a PHQ-9 score $\geq 10$ had a sensitivity of $88 \%$ and a specificity of $88 \%$ for major depression (8).

\section{Assessment of covariates}

The relationship between arthritis and depression was studied from three aspects: demographic data, healthy lifestyle, and medical history. Information on gender, age, race, education, poverty-to-income ratio (PIR), and marriage was obtained by experienced interviewers using CAPI system to conduct household population questionnaire. Body mass index (BMI) was obtained from the examination data. Race was divided into "black", "Mexican", "Hispanic" or "others" and "whites". Education was divided into "college and above" or "high school graduation" and "below high school". PIR was divided into "PIR $\geq 5$ " and "PIR $<5$ ". Marriage was divided into "living with partner", "living alone" and "married".

Healthy lifestyle was determined by investigating recreational activities and smoking habits. Moderate recreational activities were reflected in the following questionnaire: in a typical week, did you have any moderateintensity sports, fitness or recreational activities, which led to a slight increase in breathing or heart rate, such as walking fast, cycling, swimming or playing volleyball continuously for at least 10 minutes? The patient's answer to the question was "yes" or "no". Those who answered the question "have you smoked at least 100 cigarettes 
in your life?" as "yes" were determined to be smokers. Variables related to medical history, such as coronary heart disease, stroke, cancer or malignant tumor, diabetes and hypertension, etc. were collected from specific personal questions, and the patients were asked whether they had been diagnosed by doctors.

\section{Statistical analysis}

Continuous variables are represented by means and standard errors (SE), while percentages are used to represent classified variables. For continuous data, if normal distribution and homogeneity of variance are satisfied, $t$-test is used to compare between groups; otherwise, nonparametric test is used. Chi-square test is used for comparison between different types of data sets. A multivariate logistic regression models was constructed to test the relationship between depression and arthritis. In order to discuss the relationship between different conditions, three models are established in the following order. The first model (Model 1) is adjusted according to gender, age, race, BMI, PIR, education and marriage. The second model (Model 2) has made additional adjustments for moderate recreational activities and smoking. The third model (Model 3) was further adjusted according to the history of coronary heart disease, stroke, malignant tumor, diabetes and hypertension. The results are expressed by odds ratios (OR) and corresponding $95 \%$ confidence intervals $(\mathrm{CI})$. R3.6.2 is used to analyze research data. All statistical tests are double-tailed, and the significance level is set as $\alpha=0.05$.

\section{Results}

\section{Characteristics of study population}

Table 1 show the characteristics of the study population. Our final data included 25,990 patients (average age 49.3 years, male 49.1\%). Among all depression patients, 2,327 patients have major depression and other 23,663 patients have not yet reached the level of major depression. And there are 1,045 patients suffering from both major depression and arthritis. There are obvious differences in clinical features of major depression. Compared with patients without major depression, patients with major depression are more likely to be female, Hispanics, smokers, less educated, less recreational activities, PIR $<5$, patients with coronary heart disease, stroke, malignant tumor, diabetes and hypertension. Patients with major depression also have higher BMI. There is no significant difference in age between patients with and without major depression. In univariate analyses, arthritis is significantly related to major depression $(25.4 \%$ vs. $44.9 \% ; \mathrm{P}<0.001)$.

\section{Association between depression arthritis}

Table 2 show the relationship between major depression and arthritis. When the model is adjusted for other covariates, arthritis can be determined as a risk factor of major depression from OR value. When the model is only adjusted for gender, age, race, BMI, PIR, education and marriage (Model 1), the OR value is the highest among the three models $(\mathrm{OR}=2.72,95 \% \mathrm{CI}, 2.45-3.02, \mathrm{P}<0.001)$. When the model was adjusted for moderate recreational activities and smoking, the OR value decreased (Model 2) $(\mathrm{OR}=2.51,95 \% \mathrm{CI}, 2.26-2.80, \mathrm{P}<0.001)$, but remained statistically significant. When the model is further adjusted for all covariates, the OR value is the lowest (Model 3). Participants with arthritis were 2.30 times more likely to suffer from major depression than normal people (OR $=2.30,95 \%$ CI, 2.06-2.56, $\mathrm{P}<0.001$ ).

\section{Subgroup analysis}

The results of subgroup analysis are shown in Table 3 and Figure 2. In order to determine which type of arthritis has the greatest influence on major depression, we further divide arthritis into "osteoarthritis or degenerative arthritis", "rheumatoid arthritis", "psoriatic arthritis" and "other arthritis" (the control group were all other patients without arthritis). "Psoriatic arthritis" showed the highest OR value in all three models (Model $1=2.89$, Model $2=2.69$, Model $3=2.49$ ). This significant correlation exists in all models, which suggests that PsA may have the greatest influence on patients with depression.

\section{Discussion}

At present, the treatment of arthritis mainly focuses on drugs and lifestyle changes (9). However, it is difficult for patients to change their lifestyle, because the psychological characteristics of arthritis-related complications, such as depression, may lead to poor compliance with dietary counseling and exercise prevention measures (10). Depression is also related to poor compliance with medical advice for general chronic diseases (11) and rheumatological treatment. In order to address the impact of depression on the management of arthritis, it is reasonable to actively 
Table 1 Characteristics of study participants based on depression status $(\mathrm{n}=25,990)$

\begin{tabular}{|c|c|c|c|}
\hline Characteristics & No depression $(\mathrm{N}=23,663)$ & Depression $^{\mathrm{a}}(\mathrm{N}=2,327)$ & $P$ value ${ }^{b}$ \\
\hline Age, mean (SD) & $49.3(17.7)$ & $48.6(16.1)$ & 0.06 \\
\hline Race, \% & & & $<0.001$ \\
\hline White & 43.1 & 43.7 & \\
\hline Mexican & 14.4 & 14.3 & \\
\hline Hispanic & 9.6 & 13.1 & \\
\hline Other & 11.8 & 7.7 & \\
\hline BMI, mean (SD) & $29.1(6.9)$ & $31.0(8.4)$ & $<0.001$ \\
\hline Below high school & 21.5 & 34.4 & \\
\hline High school graduate & 22.9 & 24.2 & \\
\hline College or above & 55.5 & 41.4 & \\
\hline Marriage, \% & & & $<0.001$ \\
\hline Married & 52.9 & 34.1 & \\
\hline Living with partner & 8.1 & 9.9 & \\
\hline Living alone & 39 & 56 & \\
\hline Moderate recreational activities ${ }^{d}, \%$ & 42.5 & 25.8 & $<0.001$ \\
\hline History of hypertension, \% & 34.7 & 46.8 & $<0.001$ \\
\hline History of arthritis, \% & 25.4 & 44.9 & $<0.001$ \\
\hline
\end{tabular}

a , Patient Health Questionnaire (PHQ) score $\geq 10$. ${ }^{b}$, based on $t$-test for continuous variables and $\chi^{2}$ test for categorical variables. ${ }^{c}$, data are reported as percentages unless otherwise specified. ${ }^{d}$, sports, fitness, or recreational activities that cause a small increase in breathing or heart rate. ${ }^{e}$, smoking at least 100 cigarettes in lifetime. BMI, body mass index (calculated as weight in kilograms divided by height in meters squared); SD, standard deviation; PIR, ratio of family income to poverty.

screen and treat arthritis patients with depression in time.

In addition, we established three models from three aspects: demographic data, healthy lifestyle and medical history. The results showed that arthritis was an influential factor of major depression in all three models. Although previous studies have found the impacts of RA or degenerative arthritis on depression, compared with other diseases $(2-4,6)$, there is still a lack of comparison between different types of arthritis. By subdividing arthritis patients into degenerative arthritis, RA, PsA and other types of arthritis, we found that PsA has the greatest influence on major depressive symptoms. The same results appear in all three models. Previous studies have shown that psoriasis increases the risk of depression (12), and our results further 
Table 2 Multinomial logit regression analysis of depression (PHQ score $\geq 10$ ) and arthritis

\begin{tabular}{|c|c|c|c|c|c|c|}
\hline Variables & \multicolumn{2}{|c|}{ Model 1} & \multicolumn{2}{|c|}{ Model 2} & \multicolumn{2}{|c|}{ Model 3} \\
\hline History of arthritis & $2.72(2.45-3.02)$ & $<0.001$ & $2.51(2.26-2.80)$ & $<0.001$ & $2.30(2.06-2.56)$ & $<0.001$ \\
\hline Male gender & $0.64(0.58-0.70)$ & $<0.001$ & 0.57 (0.52-0.63) & $<0.001$ & $0.55(0.50-0.61)$ & $<0.001$ \\
\hline Age & $0.98(0.98-0.99)$ & $<0.001$ & $0.98(0.98-0.99)$ & $<0.001$ & $0.97(0.97-0.98)$ & $<0.001$ \\
\hline White & 1 (Reference) & & 1 (Reference) & & 1 (Reference) & \\
\hline Black & $0.76(0.68-0.86)$ & $<0.001$ & $0.81(0.72-0.91)$ & $<0.001$ & 0.78 (0.69-0.88) & $<0.001$ \\
\hline Mexican & $0.78(0.68-0.90)$ & $<0.001$ & $0.89(0.77-1.03)$ & 0.11 & $0.92(0.79-1.06)$ & 0.23 \\
\hline Hispanic & $1.12(0.97-1.29)$ & 0.13 & $1.24(1.07-1.44)$ & 0 & $1.28(1.10-1.47)$ & 0 \\
\hline $\mathrm{PIR} \geq 5$ & $0.44(0.37-0.52)$ & $<0.001$ & $0.49(0.41-0.59)$ & $<0.001$ & $0.51(0.43-0.61)$ & $<0.001$ \\
\hline \multicolumn{7}{|l|}{ Education } \\
\hline Below high school & 1 (Reference) & & 1 (Reference) & & 1 (Reference) & \\
\hline High school graduate & $0.64(0.57-0.72)$ & $<0.001$ & $0.68(0.60-0.77)$ & $<0.001$ & $0.69(0.61-0.78)$ & $<0.001$ \\
\hline College or above & $0.51(0.46-0.57)$ & $<0.001$ & $0.60(0.54-0.68)$ & $<0.001$ & $0.62(0.55-0.69)$ & $<0.001$ \\
\hline \multicolumn{7}{|l|}{ Marriage } \\
\hline Married & 1 (Reference) & & 1 (Reference) & & 1 (Reference) & \\
\hline Living with partner & $1.57(1.33-1.85)$ & $<0.001$ & $1.44(1.22-1.69)$ & $<0.001$ & $1.43(1.21-1.68)$ & $<0.001$ \\
\hline \multicolumn{3}{|c|}{ History of coronary heart disease } & & & $1.44(1.17-1.75)$ & $<0.001$ \\
\hline \multicolumn{3}{|l|}{ History of stroke } & & & $1.74(1.44-2.09)$ & $<0.001$ \\
\hline \multicolumn{3}{|c|}{ History of cancer or malignancy } & & & $1.12(0.96-1.30)$ & 0.15 \\
\hline \multicolumn{3}{|l|}{ History of diabetes } & & & $1.35(1.19-1.53)$ & $<0.001$ \\
\hline \multicolumn{3}{|l|}{ History of hypertension } & & & $1.44(1.29-1.60)$ & $<0.001$ \\
\hline
\end{tabular}

The multivariate Model 1 was adjusted for gender, age, race, BMI, PIR, education, and marriage. The multivariate Model 2 was adjusted for history of arthritis, gender, age, race, BMI, PIR, education, marriage, moderate recreational activities and smoking. The multivariate Model 3 was adjusted for history of arthritis, gender, age, race, BMI, PIR, education, marriage, moderate recreational activities, smoking, history of coronary heart disease, stroke, cancer or malignancy, diabetes, and hypertension. CI, confidence interval; OR, odds ratio; BMI, body mass index; PIR, ratio of family income to poverty.

complement the relationship between psoriasis-related diseases and major depression.

Our study found that patient with PsA had the highest risk of major depression in all three models. Metaanalysis shows that PsA patients have higher incidence of depression than the general population (4). PsA is a kind of 
Table 3 Multinomial logit regression analysis of depression (PHQ score $\geq 10$ ) and different types of arthritis

\begin{tabular}{|c|c|c|c|c|c|c|}
\hline Variables & \multicolumn{2}{|c|}{ Model 1} & \multicolumn{2}{|c|}{ Model 2} & \multicolumn{2}{|c|}{ Model 3} \\
\hline Others without arthritis & 1 (Reference) & & 1 (Reference) & & 1 (Reference) & \\
\hline Degenerative arthritis & $2.73(2.36-3.15)$ & $<0.001$ & $2.50(2.16-2.88)$ & $<0.001$ & $2.26(1.95-2.61)$ & $<0.001$ \\
\hline Rheumatoid arthritis & $2.67(2.26-3.14)$ & $<0.001$ & $2.50(2.11-2.94)$ & $<0.001$ & $2.30(1.94-2.71)$ & $<0.001$ \\
\hline
\end{tabular}

The multivariate Model 1 was adjusted for gender, age, race, BMI, PIR, education, and marriage. The multivariate Model 2 was additionally adjusted for moderate recreational activities and smoking. The multivariate Model 3 was further adjusted for history of coronary heart disease, stroke, cancer or malignancy, diabetes and hypertension. Cl, confidence interval; OR, odds ratio; BMI, body mass index; PIR, ratio of family income to poverty.

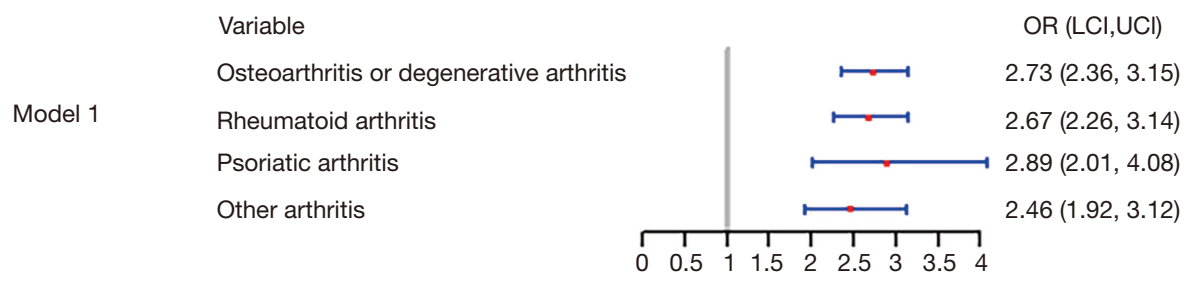

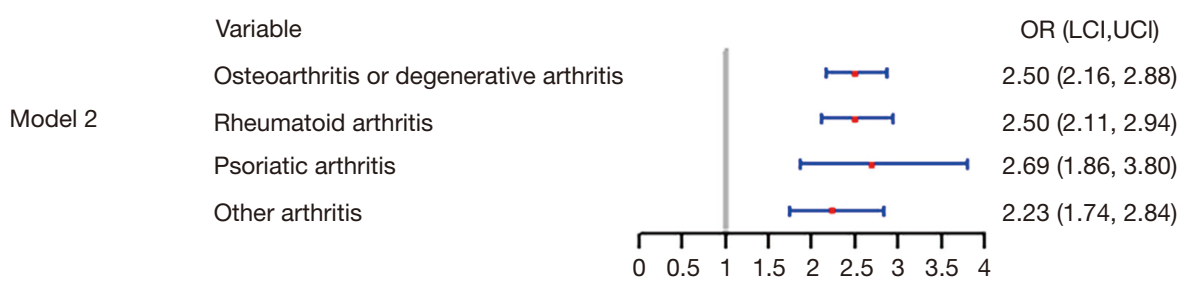

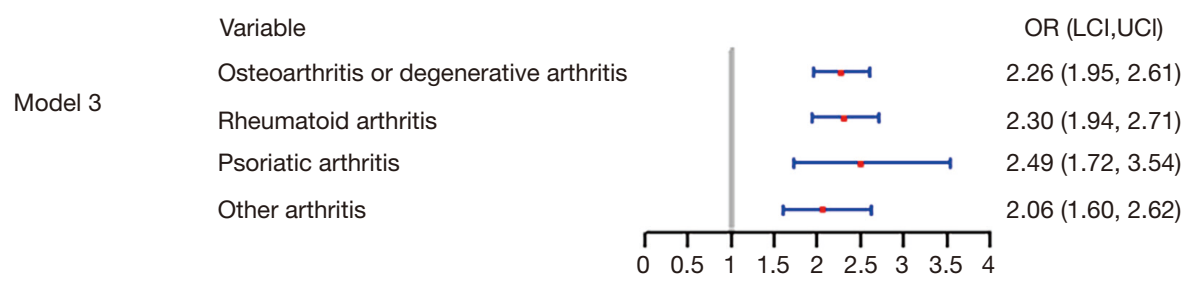

Figure 2 Forest plot of suburb analysis.

inflammatory arthritis related to psoriasis, with a prevalence rate of $0.3-1 \%$ in the general population worldwide and $6-42 \%$ in psoriasis patients (13). Patients are usually accompanied by Systemic Psoriatic Disease, including skin, joint and gastrointestinal injuries (14). Any worsening injury on the skin will bring shame to the patient, because it is the most obvious organ to be seen. Skin lesions caused by PsA will affect the appearance of patients and reduce self-acceptance and self-esteem. The typical skin lesions of PsA are hair, limbs, genitals, etc., which lead to severe humiliation of patients, especially during sexual contact. Previous studies have shown that arthritis patients are more likely to be depressed than the general population (15), and their depressive symptoms are aggravated with their sense of shame (16). PsA is also very erosive to joints, and about $20 \%$ of patients will develop destructive arthritis. Chronic pain, 
swelling, deformity and bone fusion caused by arthritis will seriously damage the self-care and mental health of patients. In addition, previous studies have revealed that psoriasis and depression shared many common causes. For example, the decrease of brain-derived neuropathy factor (BDNF) and vitamin D3 (17-19), the increase of inflammatory cytokines and the change of melatonin level (18-20). In addition, in patients with PsA, the hypothalamic-pituitaryadrenal (HPA) axis is overactive, triggering cell-mediated autoimmunity and chronic inflammation. Studies have shown that the HPA axis is also related to mental stress, and an overactive HPA is commonly seen in depression. These may explain the pathogenesis behind the higher risk of depression in patients with PsA. PsA is incurable and recurrent, which can only be controlled by early diagnosis and timely treatments. PsA, which is a recurring, longterm painful and multi-system failure, will increase the psychological burden of patients.

The complex two-way relationship between arthritis and depression has not been fully elucidated (21). However, there is evidence to support a correlation between depression and arthritis. Some pro-inflammatory cytokines such as IL-6 and tumor necrosis factor-alpha ( $\mathrm{TNF} \alpha)$ are overexpressed in arthritis, so targeting these cytokines can be used as one of the treatment methods for arthritis. And pro-inflammatory cytokines are also risk factors for the development of mental health diseases such as depression. In particular, depression will further aggravate and impair physiological responses, including effective coping behaviors, such as dysregulation of inflammation in patients with arthritis (22). Therefore, the existence of pro-inflammatory cytokines may be one of the reasonable explanations between depression and arthritis. Antidepressant therapy while treating arthritis may improve arthritis.

Since the bidirectional relationship between depression and arthritis subtypes is still unclear, further prospective cohort studies are needed to confirm our results. However, this study is still meaningful. For example, the reliability of self-reporting of various arthritis has been proved to be a satisfactory factor $(21,22)$. We collected and analyzed many high-quality data and questionnaires (PHQ-9) by trained personnel, and controlled many variables at the same time. Regardless of race/ethnicity, this abundant information will greatly extend our understanding. To our knowledge, this is the first time to use a nationally representative cohort to study the relationship between arthritis and depression.

However, there are still some limitations in this study. Firstly, major depression was self-assessed by PHQ-9, which may not be used as a standard to diagnose clinically related depression, this may result in high error rates, because not every patient knows exactly the diagnosis, better would be a Doppler check by medical report. Secondly, crosssectional design is not considered to be able to infer the causal relationship between depression and arthritis. Finally, some potential confounding variables, such as the course of depression, were not included in the data set, which might interfere with the results to some extent.

\section{Conclusions}

Although previous studies have shown that there is a certain relationship between some types of arthritis and major depression, there is still a lack of systematic and largescale cross-sectional studies, especially a lack of comparison between arthritis subgroups related to major depression. This study focused on the above correlation, and found that patients with PsA had a higher incidence of major depression than those with other types of arthritis. Our study suggests that all arthritis patients, especially PsA patients, may benefit from depression screening. Proper screening and timely treatment of depression have positive effects on the treatment and prognosis of arthritis.

\section{Acknowledgments}

Funding: This work was supported by National Natural Science Foundation of China (81774219) and Capital Foundation of Medical Development (2020-1-2121).

\section{Footnote}

Reporting Checklist: The authors have completed the STROBE reporting checklist. Available at http://dx.doi. org/10.21037/apm-21-279

Conflicts of Interest: All authors have completed the ICMJE uniform disclosure form (available at http://dx.doi. org/10.21037/apm-21-279). The authors have no conflicts of interest to declare.

Ethical Statement: The authors are accountable for all aspects of the work in ensuring that questions related to the accuracy or integrity of any part of the work are appropriately investigated and resolved. The study was conducted in accordance with the Declaration of Helsinki (as revised in 2013). 
Open Access Statement: This is an Open Access article distributed in accordance with the Creative Commons Attribution-NonCommercial-NoDerivs 4.0 International License (CC BY-NC-ND 4.0), which permits the noncommercial replication and distribution of the article with the strict proviso that no changes or edits are made and the original work is properly cited (including links to both the formal publication through the relevant DOI and the license). See: https://creativecommons.org/licenses/by-nc-nd/4.0/.

\section{References}

1. Hootman JM, Helmick CG, Barbour KE, et al. Updated Projected Prevalence of Self-Reported Doctor-Diagnosed Arthritis and Arthritis-Attributable Activity Limitation Among US Adults, 2015-2040. Arthritis Rheumatol 2016;68:1582-7.

2. Akintayo RO, Yerima A, Olaosebikan HB, et al. How much gloom is in groans? Depression and its determinants in Nigerian patients with knee osteoarthritis: a multi-center cross-sectional study. Clin Rheumatol 2019;38:1971-8.

3. Baerwald C, Manger B, Hueber A. Depression as comorbidity of rheumatoid arthritis. Z Rheumatol 2019;78:243-8.

4. Zusman EZ, Howren AM, Park JY, et al. Epidemiology of depression and anxiety in patients with psoriatic arthritis: A systematic review and meta-analysis. Semin Arthritis Rheum 2020;50:1481-8.

5. Matcham F, Rayner L, Steer S, Hotopf M. The prevalence of depression in rheumatoid arthritis: a systematic review and meta-analysis. Rheumatology (Oxford) 2013;52:2136-48.

6. Ryu E, Chamberlain A, Pendegraft R, et al. Quantifying the impact of chronic conditions on a diagnosis of major depressive disorder in adults: a cohort study using linked electronic medical records. BMC Psychiatry 2016;16:114.

7. Borrud L, Chiappa M, Burt V, et al. National Health and Nutrition Examination Survey: national youth fitness survey plan, operations, and analysis, 2012. Vital Health Stat 2 2014;(163):1-24.

8. Kroenke K, Spitzer RL, Williams JB. The PHQ-9: validity of a brief depression severity measure. J Gen Intern Med 2001;16:606-13.

9. Chalasani N, Younossi Z, Lavine JE, et al. The diagnosis and management of nonalcoholic fatty liver disease: Practice guidance from the American Association for the Study of Liver Diseases. Hepatology 2018;67:328-57.

10. Twigg S, Hensor E, Emery P, et al. Patient-reported Outcomes as Predictors of Change in Disease Activity and Disability in Early Rheumatoid Arthritis: Results from the Yorkshire Early Arthritis Register. J Rheumatol
2017;44:1331-40.

11. DiMatteo MR, Lepper HS, Croghan TW. Depression is a risk factor for noncompliance with medical treatment: meta-analysis of the effects of anxiety and depression on patient adherence. Arch Intern Med 2000;160:2101-7.

12. Cohen BE, Martires KJ, Ho RS. Psoriasis and the Risk of Depression in the US Population: National Health and Nutrition Examination Survey 2009-2012. JAMA Dermatol 2016;152:73-9.

13. Gladman DD, Antoni C, Mease P, et al. Psoriatic arthritis: epidemiology, clinical features, course, and outcome. Ann Rheum Dis 2005;64 Suppl 2:ii14-7.

14. Chimenti MS, Caso F, Alivernini S, et al. Amplifying the concept of psoriatic arthritis: The role of autoimmunity in systemic psoriatic disease. Autoimmun Rev 2019;18:565-75.

15. Devrimci-Ozguven H, Kundakci T, Kumbasar H, et al. The depression, anxiety, life satisfaction and affective expression levels in psoriasis patients. J Eur Acad Dermatol Venereol 2000;14:267-71.

16. Zięciak T, Rzepa T, Król J, et al. Stigmatization feelings and depression symptoms in psoriasis patients. Psychiatr Pol 2017;51:1153-63.

17. Brunoni AR, Lotufo PA, Sabbag C, et al. Decreased brainderived neurotrophic factor plasma levels in psoriasis patients. Braz J Med Biol Res 2015;48:711-4.

18. Slavich GM, Irwin MR. From stress to inflammation and major depressive disorder: a social signal transduction theory of depression. Psychol Bull 2014;140:774-815.

19. Tohid H, Aleem D, Jackson C. Major Depression and Psoriasis: A Psychodermatological Phenomenon. Skin Pharmacol Physiol 2016;29:220-30.

20. Rosenblat JD, Cha DS, Mansur RB, et al. Inflamed moods: a review of the interactions between inflammation and mood disorders. Prog Neuropsychopharmacol Biol Psychiatry 2014;53:23-34.

21. Martin LM, Leff M, Calonge N, et al. Validation of selfreported chronic conditions and health services in a managed care population. Am J Prev Med 2000;18:215-8.

22. Kehoe R, Wu S, Leske M, et al. Comparing self-reported and physician-reported medical history. Am J Epidemiol 1994;139:813-8.

Cite this article as: Wang MY, Li J, Peng HY, Liu J, Huang KL, Li L, Yan ZF, Zhao ZH. Patients with different types of arthritis may be at risk for major depression: results from the National Health and Nutrition Examination Survey 2007-2018. Ann Palliat Med 2021;10(5):5280-5288. doi: 10.21037/apm-21279 